\title{
From setting the distance to adjusting the focus
}

\author{
Alfred Nordmann, Hans RADDER and Gergor SchiEMANn (eds): \\ Science transformed? Debating claims of an epochal break.
}

Pittsburgh: University of Pittsburgh Press, 2011, 232 pp. \$29.95 PB

\section{Sacha Loeve}

As biotechnologies and nanotechnologies are becoming paradigmatic of nowadays science, what happened to science as we (thought we) knew it? Are we witnessing a break of an epochal character? Science Transformed? is a collection of essays that revolve around the question whether recent science hallmarks an epochal break.

The book is organized in two sections: the first one gathers essays that discuss the pertinence of the epochal break thesis as a whole, and the second provides empirical support to test the thesis in a number of fields: scientific experimentation practices and ideal-types, digital imaging media, robotics, medical research and computational modelling. The book ends on a series of 'sticking points' on which further research should focus.

While each chapter adopts a singular vantage-point to address the epochal break issue, the book as a whole offers a coherent and well-organized panorama of the problem. Each contribution nicely responds to the others and they share conceptual tools taken from common bibliographical references: 'mode-I/mode-II production of knowledge' (Gibbons et al. 1994), science/technoscience (Hottois 2005) or Paul Forman's shift from modernity-where the means of the scientific method culturally prevail over technological ends - to postmodernity - where 'technology is simply all there is' (Forman 2007: 72) and sanctions the hegemony of purely utilitarian values. The introduction reviews the current trends of concepts used to describe the changes occurring in science: 'postnormal' (Funtowicz and Ravetz 1993), 'postacademic' (Ziman 2000) or 'entrepreneurial science' propelled by a university-industry-government 'triple helix' (Etzkowitz 1983; Etzkowitz and Leydesdorff 1998). But fortunately, the book does not propose new labels. Most of its articles are actually rather critical about the pertinence of using such catchwords: They are more normative than descriptive and tend to oversimplify the history of science and engineering. Whereas most of these expressions were forged to label transformations occurring in the social and political context of science policy and funding, this volume focuses primordially on the shifts occurring in the practices and the values of scientific research. The major purpose is rather to look for the argumentative and methodological patterns that are the most appropriate to address these issues.

Amongst the contributions four different interpretations of the changes in recent science and technology are to be distinguished: (1) that there is an epochal break; (2) that today's changes are more a matter of intensification rather than rupture; (3) that other patterns than epochal break should be proposed to reflect on science's current transformations; (4) a rejection of the epochal break thesis.

\footnotetext{
${ }^{1}$ S. Loeve $(\bowtie)$

Centre d'Etude des Techniques, des Connaissances et des Pratiques (CETCOPRA),

Université Paris 1 Sorbonne, 17 rue de la Sorbonne, 75005 Paris, France

e-mail: sacha.loeve@univ-paris1.fr
} 
(1) The epochal break thesis to be discussed in the volume is articulated by Alfred Nordmann, who pleads for the recognition of a shift from the scientific enterprise to the regime of technoscience. With technoscience, he contends, it becomes neither possible nor required to discriminate between practical intervention in the world and theoretical representation of the world. Understanding and control are indistinguishable.

In the first part of the book (where the burden of proof is heavier), most authors actually dispute this view. It is mostly in the second part that we find endorsements of Nordmann's thesis. Angela Krewani, for instance, claims that contemporary imaging practices have "done away with the implied distance between image and viewer" to bring about "an ongoing conflation of viewer, apparatus, and image" (p. 156).

In their essay on experimentation, Astrid Schwarz and Wolfgang Krohn also provide support to the epochal break thesis by testifying to a shift from the 'laboratory ideal' to the 'field ideal' of experimentation. Such a shift, they argue, means that the old Baconian science-society contract is being replaced with a new one: scientific experiments are no more conducted only in the laboratory defined as a socially confined space; they are no more affecting society's choice of options only by those originating from already approved scientific knowledge. The new science-society contract is defined as a process of 'social experimentation' engaging society as a giant laboratory where 'real-world simulations' are performed.

That there has been an epochal break in biomedical science is also acknowledged by James Robert Brown. In his view, the feeling that the 'good old days' are over makes the shift obvious. Significant accomplishments for improving the quality of our lives are behind us (he takes the case of anti-hypertension drugs). The rule now is 'follow the money'. However, while Brown warns us about the erosion of scientific quality under the influence of commercial interests, he is not willing to generalize his diagnosis to all medical science, and even less to all science: "People investigating the role of diet and exercise on depression resemble past researchers, just as physicists do" (p. 186).

(2) Some chapters argue for an incremental change rather than an epochal break. For instance Valerie Hanson in her study of digital imaging claims that though the transition from analog to digital practices may have induced a lot of incremental changes, it has not brought about a revolution in knowledge production and communication.

An interesting intensification pattern is described by Martin Carrier. To him, the real technoscientific turn lays behind us: it was the scientific revolution. Modern science, he claims, has always pursued the technoscientific ambition of making and remaking the world. Consequently, instead of a new rupture, we are rather witnessing the concretization and amplification of the effects of an epochal break that occurred centuries ago, and which delivers only now its bunch of promises and problems. As to the alleged replacement of understanding by control involved in Nordmann's argument, Carrier considers it to be an epistemic strategy doomed to abortion and promised to an inglorious ending because of the lack of robustness and sustainability that its generalization would entail (a claim which is finely disputed by Ann Johnson and Johannes Lenhard's contribution on computational modelling: by their very opaqueness and versatility, computational models, they argue, concretize a new culture of prediction that deliberately assumes missing sustainability). However, Carrier ends by remarking that a new 'technoscientific turn' might be found at the level of research objects' ontology: Technoscientific objects like chlorofluoro- 
carbons, ferromagnetic materials, liquid crystals, light-emitting diodes or nanoobjects are not discovered in nature-in-self; they are simultaneously created by humans and explored like natural objects of they own right. But how uncanny these technoscientific objects might be ontologically, methodologically, Carrier maintains, they still need scientific knowledge in order to be controlled and used.

(3) Other chapters develop tentative alternative interpretations of what is going on in science: Hans Radder, like Carrier, considers interventionist ambitions as a perennial trait of modern science. He also finds the model of knowledge-as-control highly questionable regarding its epistemic validity. Instead of an epochal rupture between science and technoscience, Radder bases his diagnostic on the existence of novel and nonlocal patterns: the commoditization of academic culture (and the correlated erosion of its critical ethos); the increasing prevalence of external norms of validity (economic competitiveness and social acceptability).

As to Andrew Jamison, he points out a transitional process of 'changing contexts of science and technology' from disciplinary 'mode-1' little science (before WWII) to multidisciplinary 'mode 1.5 ' big science (1940-1960s), and then to a 'mode-2' transdisciplinary technoscience (1970s-onward). But it does not mean that current technoscience opens up a new epoch. It could well be just a transition towards a 'mode-3' research culture: a more desirable synthesis of disciplinary science and commercial technoscience.

Chunglin Kwa makes the case for a pluralistic interpretation. Instead of accounting for today's technoscience in terms of shifting relations between two entities (science/technology) he suggests a constellation of multiple scientific styles (Crombie 1994): deductive, experimental, taxonomical, analogical-hypothetical, statistical, historical-evolutionary. He then considers technology as a full-fledged style resulting from various alliances with other scientific styles, and taking different forms: theoretical engineering research, technological knowledge, invention, design, transfer or application from science, spatial thinking, analogical thinking, etc. Consequently the configurations are too diverse to give way for the prevalence of a single one over the others. Moreover, each partner maintains its identity, so as we are not witnessing an eclipse on the part of science.

(4) Most chapters in the book's first part argue against the epochal break thesis. For Gregor Schiemann, the transition from medieval to early modern science has been perceived as an epochal break, only a posteriori, because a time lapse is required to understand the global significance of a process. "We always remain involved in the events of our times (p. 37)". For Schiemann, the departure from early modern epistemic norms of universality, necessity, and truth is a process that started in the nineteenth century and about which no one knows if it has come to an end. Schiemann also argues against the blurring of the divide between science and technology, nature and artefact, allegedly characteristic of today's technoscience. Early modern science already disposed of the ancient distinction between physis and technè. The nature/technology divide changes, but it does not vanish. Nano-objects or the OncoMouse, for instance, are not purely human artefacts, they are still made of natural parts or are still organisms belonging to nature (though seriously damaged by humans).

Cyrus Mody is even more sceptical. He picks out various examples in the history of science and engineering with the characteristic features of current technoscience: nuclear devices, game theory, Langmuir's light bulb, Thompson's telegraph and Galileo's workshop. None of them was empirical engineering or applied science. All 
of them already blurred the traditional frontiers of scientific disciplines and ethos. Therefore, Mody argues against any grand claims about epochal breaks: "There might be breaks, but they don't seem very epochal, unless an epoch only lasts a generation or so"' (p. 62).

Finally, Mieke Boon and Tarja Knuutilla want to 'break up with the epochal break'. On the basis of the epistemology of models, they argue that engineering science offers good rationale for breaking up, respectively, with the representing/intervening and basic/applied divides that are mistakenly taken as criteria of classic modern science or mode-I research.

"No one can leap beyond his own age", said Hegel. For sure, the only factual answer to the epochal break problem is that it cannot be settled as a matter of fact: A historical diagnosis is never neutral; it is an exercise engaging values within and from the present. Most contributions to Science Transformed? against the epochal break thesis actually warn us about the rhetoric that exploits the epochal break for legitimizing shortterm reorientations of the scientific enterprise: "Announcement of epochal breaks are often interested and have real consequences" (p. 10). Consequently, supporting the epochal break thesis would be tantamount to amplifying and supporting trends that are judged undesirable.

Nordmann, by contrast, argues that technoscience is insensitive to historical selfunderstanding, so that is precisely denying the epochal break which amounts to endorsing the regime of technoscience:

From the point of view of science and how it understands itself, hardly anything could be as dramatic as the shift to a technoscientific mode of research. From the point of view of technoscience, in contrast, the whole history of science and engineering research has always been technoscientific. (...) Those who deny the epochal break thesis have happily settled into the age of technoscience (p. 20).

For Nordmann, seeing or not seeing an epochal break is therefore a matter of setting the proper distance: If one remains too close, immersed in the multiplicity of singular practices, any pretension to identify some overall shift will necessarily seem illegitimate. One should thus step back and take some distance, but not the too detached distance of positivist, analytical or purely formal epistemologies of science-rather a kind of 'engaged distance' - far enough to identify global patterns, close enough to be valuesensitive.

Here is a volume displaying a wide spectrum of arguments pro and contra the notion of epochal break. However, despite all its richness the way the book frames the question has its limits. All contributors seem to agree at least on one point: whether there is a shift towards technoscience or not, technoscience is not something highly desirable. Accordingly technoscience is essentially characterized in a negative way as: blurring of distinctions, nonsustainalibility and erosion of scientific ethos. What about the various changes that matter without being epochal, or traits of novelty that might be 'slipping under the radar' (p. 144)? The ontological specificity of technoscientific objects pointed out by Carrier and pinpointed as a 'sticking point' in Radder's epilogue is too quickly discarded in favour of an interpretation essentially based on epistemological and historiographical considerations. Perhaps setting the proper distance is less important than identifying the 'right' one, 'adjusting the focus' to 'do justice' or to 'render visible' what deserves to be rendered visible.

Another limitation of the book is that it seeks to capture a transformation on the basis of fixed definitions of what is being transformed. Let's define science, and then we shall see how it has changed. Bergson's criticism of all attempts aiming at reconstructing 
an evolutionary process by starting from the processed or by combining processed elements could be relevant here. Similarly, one cannot start from the transformedscience-to account for its transformations. Indeed it is not an entirely illegitimate inference but it will result in a diagnosis, not in a genesis. A diagnosis is about the normal and the pathological. It cannot do otherwise than fixing some characters in order to determine what has changed. Alternatively, a genesis would not presuppose a definition of science as the 'subject of change' and rather consider science or technoscience 'in transformation' and follow their process of stabilization in objects.

Such is the perspective explored in the project GOTO 'The Genesis and Ontology of Technoscientific Objects' conducted by Bernadette Bensaude-Vincent, Sacha Loeve, Alfred Nordmann and Astrid Schwarz. Instead of discussing whether technoscience is an historical rupture or business as usual, the GOTO project assumes that: (1) Technoscience is a regime of research that is now dominant, although it can be instantiated in the past centuries; (2) At the ontological level, technoscience brings a specific mode of existence of objects (and of our co-existence with them) which is distinct from the mode of existence of scientific objects (Bensaude-Vincent et al. 2011) as well as from the mode of existence of technical objects; (3) Instead of arguing pro or contra technoscience, the main task is to appraise and tame alternative modes of togetherness, worldliness and coexistence with objects.

\section{References}

Bensaude-Vincent, Bernadette, Sacha Loeve, Alfred Nordmann, and Astrid Schwarz. 2011. Matters of interest: The objects of research in science and technoscience. Journal for General Philosophy of Science 42: 365-383.

Crombie, Aslistair C. 1994. Styles of scientific thinking in the European tradition: The history of argument and explanation especially in the mathematical and biomedical sciences and arts, vol. 3. London: Duckworth.

Etzkowitz, Henri. 1983. Entrepreneurial scientists and entrepreneurial universities in American academic science. Minerva 21: 198-233.

Etzkowitz, Henri, and Loet Leydesdorff. 1998. The endless transition: A triple helix of university-industry-government relations. Minerva 36: 271-288.

Forman, Paul. 2007. The primacy of science in modernity, of technology in postmodernity, and of ideology in the history of technology. History and Technology 23: $1-152$.

Funtowicz, Silvio O., and Jerome R. Ravetz. 1993. The emergence of post-normal science. In Science, politics and morality: Scientific uncertainty and decision making, ed. René von Schomberg, 85-183. Dordrecht: Kluwer.

Gibbons, Michael, Camille Limoges, Helga Nowotny, Simon Schwartzmann, Peter Scott, and Martin Trow. 1994. The new production of knowledge: The dynamics of science and research in contemporary societies. London: Sage.

Hottois, Gilbert. 2005. Technoscience. In Encyclopedia of science, technology and ethics, vol. 4, ed. Carl Mitcham. New York: Macmillan Reference.

Ziman, John. 2000. Real science: What it is, and what it means?. Cambridge: Cambridge University Press. 